\title{
The generation effect: Dissociating enhanced item memory and disrupted order memory
}

\author{
NEIL W. MULLIGAN \\ Southern Methodist University, Dallas, Texas
}

\begin{abstract}
Generating stimuli at encoding typically improves memory for occurrence (item memory) but might disrupt memory for order. In three experiments, the relationship between generation and order memory was examined by using familiar stimuli, which give rise to the standard generation advantage in item memory, and unfamiliar stimuli, which do not. The participants generated or read words and nonwords in Experiments 1 and 2 and familiar and unfamiliar word compounds in Experiment 3. For the familiar stimuli, generation enhanced item memory (as measured by recognition) but disrupted performance on the order-reconstruction test. For the unfamiliar stimuli, generation produced no recognition advantage and yet persisted in disrupting order reconstruction. Thus, the positive effects of generation on item memory were dissociated from its negative impact on order memory.
\end{abstract}

Self-generated information is often better remembered than information that is merely perceived, a phenomenon known as the generation effect (Slamecka \& Graf, 1978). In a typical study of this effect, participants generate some study words (perhaps from an antonym or a word fragment) and read others. On a later memory test, the generated items usually produce better performance. The generation effect has been observed with a variety of materials (e.g., single and compound words, sentences, abbreviations, numbers), in which a variety of generation tasks (e.g., generation from antonyms, semantic associates, rhymes, anagrams, word fragments, second-language translations, definitions have been used), and on a variety of memory tests (e.g., free recall, cued recall, recognition, comprehension; see Greene, 1992; Mulligan, 2001a, for a review).

Despite the impressive generality of this effect, generation does not always enhance memory and may even disrupt certain aspects of performance. For example, when the generation manipulation is implemented in a betweensubjects or pure-list design, generation typically does not enhance recall (e.g., Hirshman \& Bjork, 1988; Slamecka \& Katsaiti, 1987; although, see Mulligan, 2001a, for evidence that a between-subjects generation effect emerges over repeated recall tests). Under some conditions, purelist designs can even produce a negative generation effect in which the read condition produces superior recall (e.g., Nairne, Reigler, \& Serra, 1991; Steffens \& Erdfelder, 1998). A second negative generation effect, especially important in the present context, has been reported for tests of order

This research was partially supported by Grant 1-R03-MH61324-01 from NIMH. Correspondence should be addressed to N. Mulligan, Department of Psychology, University of North Carolina, Chapel Hill, NC 27599-3270 (e-mail: nmulligan@unc.edu). memory; although generation may enhance memory for the occurrence of a stimulus, it can disrupt memory for serial-order information (e.g., Burns, 1996; Burns, Curti, \& Lavin, 1993; Nairne et al., 1991; Serra \& Nairne, 1993; cf. Kelley \& Nairne, 2001). ${ }^{1}$

A prominent account argues that the effects of generation on item and order information are central to our understanding the effects of generation on memory in general (Burns, 1996; Burns et al., 1993; DeLosh \& McDaniel, 1996; Nairne et al., 1991; Serra \& Nairne, 1993). This view is related to multifactor accounts of generation, which propose that generation enhances item but disrupts interitem relational processing (e.g., deWinstanley, Bjork, \& Bjork, 1996; Hirshman \& Bjork, 1988; Hunt \& McDaniel, 1993; McDaniel, Wadill, \& Einstein, 1988; Steffens \& Erdfelder, 1998). Item information refers to features of an item that are unique or distinctive, whereas relational information refers to associations between items within an encoding context, including those that underlie serialorder information (e.g., Hunt \& McDaniel, 1993; McDaniel, DeLosh, \& Merritt, 2000). Given the importance of both item and interitem relational information to free recall (Hunt \& McDaniel, 1993), this view accounts for the dependence of generation effects on experimental design. According to this view, when generate items are presented in a pure list, disruption of order and interitem encoding is list-wide and detracts from the typical generation effect in recall, causing generation effects to disappear or even reverse (e.g., Hirshman \& Bjork, 1988; Kinoshita, 1989; Slamecka \& Katsaiti, 1987; Steffens \& Erdfelder, 1998), even though the generation advantage persists in recognition, a test with little reliance on interitem processing (e.g., Hunt \& McDaniel, 1993; Kinoshita, 1989). In mixed lists, the disruption of intertarget processing affects read and generate items equally, which in turn per- 
mits the superior item encoding in the generate condition to produce a recall advantage (Hunt \& McDaniel, 1993; Nairne et al., 1991).

A number of variables besides generation have been proposed to enhance item encoding (see Hunt \& McDaniel, 1993). Interestingly, a number of these produce a disruption to order encoding similar to that produced by generation. These manipulations include bizarre versus common sentences (McDaniel, Einstein, DeLosh, May, \& Brady, 1995), enacted versus observed events (Engelkamp \& Dehn, 2000), low- versus high-frequency words (DeLosh \& McDaniel, 1996), and words presented with or without perceptual interference (Mulligan, 1999). As DeLosh and McDaniel have noted, these manipulations typically contrast a relatively unusual encoding condition(e.g., the generate condition, bizarre sentences, low-frequency words) with a more common encoding condition (e.g., the read condition, common sentences, high-frequency words), leading to the suggestion that the unusual condition attracts greater attention to item characteristics at the expense of processing order and relational information (DeLosh \& McDaniel, 1996; Engelkamp \& Dehn, 2000; Nairne et al., 1991; Serra \& Nairne, 1993).

It is theoretically important to study memory for order because disrupted memory for order might be causally related to superior item memory in the unusual encoding condition (DeLosh \& McDaniel, 1996; Engelkamp \& Dehn, 2000; Nairne et al., 1991; Serra \& Nairne, 1993; see Greene, Thepar, \& Westerman, 1998, for discussion). However, there are reasons to question whether disrupted order encoding and enhanced item encoding are inexorably linked in this set of encoding manipulations. Recent research on the perceptual-interference effect motivates this question (Mulligan, 2000a). The perceptualinterference effect is observed when some study words are presented very briefly (e.g., $100 \mathrm{msec}$ ) and then are backward masked (the perceptual-interference condition), whereas others are presented for a longer period (e.g., $2.5 \mathrm{sec}$; the intact condition). The perceptual-interference condition typically leads to better performance on tests of item occurrence, such as recognition and free and cued recall (e.g., Hirshman \& Mulligan, 1991; Mulligan, 1996, 1998; Nairne, 1988). However, as noted above and similar to generation, perceptual interference disrupts memory on tests of order memory (Mulligan, 1999, 2000a). In addition, the perceptual interference effect depends on experimental design; the perceptual interference manipulation produces strong effects on recall in within-subjects (or mixed-list) designs but typically not in between-subjects (or pure-list) designs (e.g., Hirshman \& Mulligan, 1991; Mulligan, 1999). When order information is an important determinant of recall, both the generation and perceptualinterference effects can reverse, with the intact or read condition leading to better recall (Mulligan, 1999; Nairne et al., 1991; Serra \& Nairne, 1993). Finally, generation and perceptual interference produce similar results in studies of hypermnesia using multiple recall tests (Mulligan, 2000b, 2001a).
Given the similarities between the effects of generation and perceptual interference, the recent results of Mulligan (2000a) are suggestive of the relationship between generation and order memory. Mulligan (2000a) used a limiting condition of the perceptual-interference effect (i.e., a version of the perceptual-interference manipulation that does not give rise to the typical advantage in item memory) to examine the relationship between perceptual interference and order. Delaying the onset of the mask in the perceptualinterference condition (thus increasing the presentation time of words) produces such a limiting condition. When the mask is delayed to a point at which it no longer interferes with word perception (e.g., $266 \mathrm{msec}$ ), the typical perceptual-identification effect in item memory is not found in recall (Hirshman, Trembath, \& Mulligan, 1994) or recognition (Mulligan, 2000a). Study items were presented under three conditions - the standard perceptualinterference condition (with a 100-msec mask), a delayed masking condition (with a 266-msec mask), and an intact control condition (with no mask). On a later recognition test, the standard perceptual-interference condition produced better performance than either the delayed mask or the intact condition, which did not differ from one another. However, on the order-reconstruction task, the standard and delayed conditions produced equivalently worse performances than did the intact condition. Thus, the standard perceptual-interference condition enhanced item memory but disrupted order memory, whereas the delayed masking condition had no effect on item memory but persisted in disrupting order memory. Given the similarities between the perceptual-interference and generation effects, these results raise the possibility that the positive effects of generation on item memory can likewise be dissociated from its negative impact on order memory.

In the following experiments, the relationship between generation and order memory was investigated by examining limiting conditions of the generation effect. One important determinant of the generation effect is the type of materials used. The generation effect in item memory is typically not obtained with unfamiliar materials, such as nonwords and unfamiliar word compounds, prompting claims that the generation effect is obtained only for stimuli with preexisting semantic and/or lexical representations (e.g., Gardiner, Gregg, \& Hampton, 1988; Gardiner \& Hampton, 1985; McElroy \& Slamecka, 1982; Nairne, Pusen, \& Widner, 1985; Payne, Neely, \& Burns, 1986; cf. Johns \& Swanson, 1988).

In the present studies, the methodology of Nairne et al. (1991; see also DeLosh \& McDaniel, 1996; Engelkamp \& Dehn, 2000; Mulligan, 1999) was used to compare the effects of generating familiar versus unfamiliar stimuli on item and order memory. In Experiments 1 and 2, the materials were words and nonwords. In Experiment 3, familiar and unfamiliar word compounds were employed. In Experiments 1 and 3, the participants were presented with multiple study-test trials in which a short study list was presented in either the generate or read condition. Following $30 \mathrm{sec}$ of distraction, the participant was presented 
with either a test of order memory (the order reconstruction test) or a row of asterisks indicating no test on the current trial. The untested lists were included in an end-ofsession recognition test (a measure of item memory). In Experiment 2, it was verified that generation's disruption of order memory occurs even when participants are not solely focused on order information during encoding.

\section{EXPERIMENT 1}

\section{Method}

Participants. Thirty-two undergraduates at Southern Methodist University participated in exchange for course credit in psychology courses.

Materials and Design. Item type (word vs. nonword), encoding condition (read vs. generate), and test type (order reconstruction vs. recognition) were varied within subjects. The stimulus materials consisted of 104 words and 104 nonwords. The words were common four- and five-letter words with Kučera-Francis (1967) frequencies between 100 and 500 . The nonwords were based on a separate set of words similar in length and frequency to the target words. For each of these words, one or two letters were replaced to produce a pronounceable nonword (e.g., trass, meep, lart). Care was taken so that none of the nonwords were homophonic with real words. The items were randomly divided into 26 study lists of 8 words or nonwords each. All items within a study list were of the same type (either words or nonwords) and were presented in the same encoding condition (either read or generate). Item type and encoding condition varied across study lists. The order of the study lists was random, with the constraint that no more than 2 consecutive study lists could be of the same item type or encoding condition.

The experiment consisted of 26 study-test blocks. The first two blocks were practice and were excluded from the analysis. Of the remaining 24 critical blocks, the study lists were evenly split between the two item types and two encoding conditions, manipulated between lists. Each study list was followed by either an order-reconstruction test or by a line of asterisks (signifying no test in this block; these study items were tested in the end-of-session recognition test). The study lists were assigned to test conditions so that 3 blocks appeared in each of the item type, encoding condition, and test combinations (e.g., word-read-order reconstruction, word-read-asterisks, wordgenerate-order reconstruction, etc.). Four sets of study-test blocks were created so that, across sets, each study list appeared in each encoding condition-test combination. Equal numbers of participants were presented with each of the four stimulus sets.

The end-of-session recognition test consisted of 96 studied items (24 of each of the item-type-encoding condition combinations) from the untested blocks, randomly intermixed with 48 new items ( 24 words and 24 nonwords). The new words and nonwords were similar to their studied counterparts. The new words were common four- and five-letter words. The new nonwords were created in the same way as the studied nonwords.

Procedure. The participants were tested individually. They were informed that the experiment consisted of a series of study-test blocks, each block composed of a study list, a distracter task, and, in half the blocks, a memory test. The participants were informed that some study lists comprised words and that other lists comprised letter strings that did not form words (i.e., nonwords). The participants were further informed that on some trials, they were to read the study items (and copy them onto an answer sheet), and on other trials, they were to generate the study item by reversing the first two letters. They were informed that on generate trials, the first two letters would be underlined. Examples of a word and a nonword, in both the read and the generate conditions, were provided.

Prior to each study list, an answer sheet was placed in front of the participant. The study list began with a BEGINNING OF LIST mes- sage displayed in the center of the computer screen for $2 \mathrm{sec}$. Next, the series of eight study items were presented. In the generate condition, the first two letters of the study item were underlined. In the read condition, the study item was presented with all letters in the correct position and with no underlined letters. Each study item was presented for $5 \mathrm{sec}$ followed by a blank screen for $300 \mathrm{msec}$. Prior to the presentation of the first item, the participants did not know the item type or encoding condition of the list. After the eighth study trial, an END OF LIST message was displayed for $3 \mathrm{sec}$. At this point, the experimenter removed the study answer sheet.

The distractor task followed the study list. This task consisted of categorizing as odd or even a series of digits (randomly chosen from 1-9). Each digit was presented for 1,000 msec followed by a blank screen for $100 \mathrm{msec}$. The participants were instructed to indicate whether the digit was odd or even by pressing "o" or "e" on the computer keyboard. The distracter task continued for 27 trials, lasting for $29.7 \mathrm{sec}$.

On half the blocks, the distractor task was followed by the orderreconstruction test, in which all eight study words were presented simultaneously on the computer screen in a new, random order. The participants were instructed to reorder the words to recreate the original order of presentation in the study list. The participants were provided with a test sheet consisting of a set of blank spaces, numbered 1-8. The participants were asked to write the words on the sheet, using each word once. The words stayed on the computer screen until the participant completed the test, whereupon the test sheet was removed and the participant pressed the space bar to continue to the next study list. On the other half of the blocks, the distractor task was followed by a line of asterisks on the computer screen. The asterisks remained on the screen for $5 \mathrm{sec}$, after which a message appeared prompting the participant to hit the space bar to continue with the next list. During presentation of the study items, the participants did not know whether the list would be followed by order reconstruction or by asterisks.

After the last study-test block, the participants received the endof-session recognition test. They were told that they would be tested for their memory of the words and nonwords that they had read and generated in the earlier part of the experiment. The participants were told that they would see a series of words and nonwords on the computer screen. They were asked to indicate which had been presented earlier by typing the " $y$ " key for old words and nonwords and the " $n$ " key for new words and nonwords. It was made clear that the test items would be presented in the form in which they had been written down on the answer sheet- that is, with the first two letters reversed for the generated items or in the originally presented form for the read items. Each test word was presented individually and remained on the screen until the participant responded.

\section{Results and Discussion}

The participants correctly generated $99.6 \%$ and $98.5 \%$ of the study words and nonwords, respectively. The corresponding means for the read items were $100 \%$ and $99.4 \%$. Although study performance was quite high in this and subsequent experiments, analyses were performed on the test data both conditionalized on correct performance at study (i.e., excluding items that were not generated correctly) and unconditionalized. Not surprisingly, both sets of analyses led to the same conclusions, and only the unconditionalized test data are reported.

First considered are the results of the recognition test, a traditional measure of item memory (Table 1). A preliminary analysis indicated that false alarm rates did not differ between words and nonwords $(F<1)$. $d^{\prime}$ recognition accuracy was submitted to a $2 \times 2$ analysis of variance (ANOVA), with item type and encoding condition as factors. The analysis produced a significant item type $\times$ en- 
Table 1

Experiment 1: Proportion of Items Judged as Old on the Recognition Memory Test as a Function of Encoding Condition and Item Type

\begin{tabular}{lccc}
\hline & \multicolumn{3}{c}{ Encoding Condition } \\
\cline { 2 - 4 } Item Type & Read & Generate & New \\
\hline Words & .66 & .74 & .30 \\
Nonwords & .67 & .65 & .30 \\
\hline
\end{tabular}

coding condition interaction $\left[F(1,31)=17.39, M S_{\mathrm{e}}=\right.$ $0.0619]$. The two main effects were not significant ( $p \mathrm{~s}>$ .10). Planned contrasts indicated an effect of encoding condition for words $[t(31)=3.33$; mean $d \mathrm{~s}=1.08$ and 1.38 for the read and generate conditions, respectively] but not for nonwords $[t(31)<1$; mean $d \mathrm{~s}=1.06$ and 0.99 for the read and generate conditions, respectively]. The same pattern of results was obtained by using corrected hits (hits - false alarms) as the measure of accuracy. Thus, the present study replicates prior research with respect to item recognition. A robust generation effect was found for words but no effect was found for nonwords (e.g., McElroy \& Slamecka, 1982; Nairne et al., 1985; Payne et al., 1986).

Accuracy in the order-reconstruction test was measured by the proportion of study words allocated to their original position (Figure 1). Here, the central result is that generation disrupted order memory for both words and nonwords. The proportions correct were submitted to a $2 \times$ $2 \times 8$ ANOVA, with item type, encoding condition, and serial position as factors. The analysis revealed significant main effects of item type $\left[F(1,31)=14.12, M S_{\mathrm{e}}=0.4244\right]$, encoding condition $\left[F(1,31)=7.71, M S_{\mathrm{e}}=0.2344\right]$, and serial position $\left[F(7,217)=10.13, M S_{\mathrm{e}}=0.0588\right]$. None of the interactions were significant (all $p s>.15$ ).

The effect of item type indicates that words produced better performance than did nonwords (Mulligan, 2001b). The significant effect of serial position replicates earlier results (e.g., Nairne et al., 1991; Mulligan, 1999), indicating a primacy effect and an approximately bow-shaped serial position curve. The most important finding is the main effect of encoding condition, which indicates better order memory for the read than for the generate items. Averaging over serial positions, the mean proportions correct for read and generated words were .64 and .56, respectively. The corresponding means for nonwords were .49 and .40. In the case of words, this result replicates prior research indicating that generation disrupts order encoding (e.g., Nairne et al., 1991; Serra \& Nairne, 1993). Critically, this result also obtains for nonwords. Furthermore, the nonsignificant item type $\times$ encoding condition interaction $(F<1.0)$ indicates that the size of the negative generation effect in order memory is comparable for words and nonwords (and is, in fact, numerically larger for nonwords). Because this negative generation effect for nonwords is a critical finding, it is important to verify its presence in the nonword condition considered by itself (rather than merely infer its presence from the main effect and nonsignificant interaction of the prior analysis). Consistent with the main analysis, there was a significant neg-

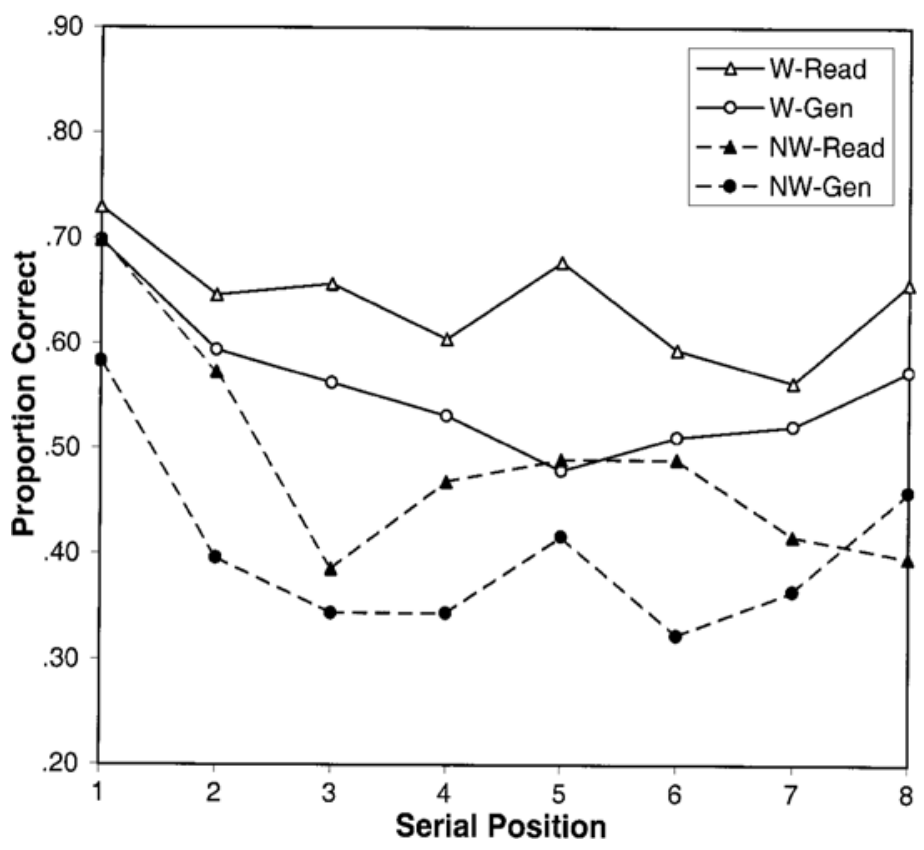

Figure 1. Experiment 1: Mean order reconstruction scores as a function of item type, encoding condition, and serial position $(\mathrm{W}=$ word, $\mathrm{NW}=$ nonword, Gen = generate condition). 
ative generation effect in order memory when the analysis was restricted to nonwords $\left[F(1,31)=5.20, M S_{\mathrm{e}}=\right.$ $0.1822]$.

As earlier studies have found (e.g., Nairne et al., 1991; Serra \& Nairne, 1993), generating words enhances item memory (as measured by recognition) and disrupts order memory (as measured by order reconstruction). In addition, generating nonwords produces no measurable effect on item encoding but persists in disrupting order encoding. The dissociation of the positive (item) and negative (order) effects of generation is theoretically important. However, this conclusion rests partly on a null result: Encoding condition did not affect recognition for nonwords. Consequently, one must consider whether this null effect is an artifact of low power. This is unlikely for several reasons. First, generation produced as large an effect on order memory for nonwords as for words, indicating that generating nonwords is not simply a weaker version of the generate manipulation with words. Second, if the null effect of generation with nonwords is a power artifact, one would expect a numerical trend favoring the generate condition on the recognition test. In fact, recognition accuracy is numerically less for generated than for read nonwords, opposite of the effect found with words. Finally, a power analysis indicates substantial power to detect a generation effect on recognition with nonwords. A to-be-detected effect size was estimated from the effect of generation on recognition memory produced by words $(d=.834$, based on Cohen, 1988, Equation 2.3.9). With $n=32$ and $\alpha=$ .05 (one-tailed), the power to detect a difference between generated and read nonwords on recognition was .94. This indicates substantial power to detect a generation effect in recognition for nonwords of the size produced by words.

\section{EXPERIMENT 2}

In Experiment 1, it was demonstrated that generating words or nonwords disrupts order memory. It should be noted, however, that during the study-test blocks of Experiment 1 , the participants were tested only on order reconstruction, which may have induced them to focus exclusively on order information during encoding. Experiment 2 was conducted to determine whether the orderdisrupting effects of generation persist when participants are led to expect order tests and free recall tests. In this experiment, half the study lists were followed by order reconstruction and half by free recall tests (no end-of-session recognition test was used).

In Experiment 2, an additional implication of the orderdisrupting effects of generation was also tested. Order information is an especially important determinant of free recall in this paradigm (e.g., DeLosh \& McDaniel, 1996; Nairne et al., 1991; Serra \& Nairne, 1993), presumably because participants adopt a serial-order retrieval strategy (Nairne et al., 1991). Consequently, to the extent that generation disrupts order encoding, it should produce worse recall than the read condition for both words and nonwords. Prior research confirms this expectation for words (e.g., Nairne et al., 1991). The present experiment determined whether the result extends to nonwords.

\section{Method}

Participants. Forty-nine undergraduates at Southern Methodist University participated in exchange for course credit in psychology courses.

Materials, Design, and Procedure. Item type (word vs. nonword), encoding condition (read vs. generate), and test type (order reconstruction vs. free recall) were varied within subjects. The materials and procedures were the same as in Experiment 1 with the following changes. First, the untested (asterisks) blocks of Experiment 1 were replaced with free recall tests. On the test portion of these blocks, the participants were instructed to recall the items from the study list and write them down in the order in which they were recalled. Second, there was no end-of-session recognition test.

\section{Results and Discussion}

One of the participants generated fewer than $60 \%$ of the nonwords and was replaced, leaving an effective sample size of 48 . The participants correctly generated $99.7 \%$ and $99.2 \%$ of the study words and nonwords, respectively. All of the read items were correctly copied.

Proportions correct on the order reconstruction and free recall tests (Figures 2 and 3 ) were submitted to separate $2 \times 2 \times 8$ ANOVAs, with item type, encoding condition, and serial position as factors. For order reconstruction, the analysis revealed three significant effects: (1) encoding condition $\left[F(1,47)=20.27, M S_{\mathrm{e}}=0.1343\right]$, which indicates better order memory for the read than for the generate condition, (2) item type $\left[F(1,47)=83.44, M S_{\mathrm{e}}=\right.$ 0.1569 ], which indicates better order memory for words than for nonwords, and (3) serial position $[F(7,329)=$ $\left.44.64, M S_{\mathrm{e}}=0.0599\right]$. None of the interactions were significant (all $p s>.10$ ). The results of order reconstruction replicate the results of Experiment 1. Generation disrupted order memory for both words and nonwords. Averaged over serial positions, the mean proportions correct for read and generated words were .72 and .63 , respectively. The corresponding means for nonwords were .56 and .48. An analysis restricted to the nonwords also indicated a significant negative generation effect $[F(1,47)=$ $\left.5.64, M S_{\mathrm{e}}=0.2218\right]$. In the present study, the participants expected either order or recall tests. Thus the results of Experiment 1 generalize to conditions under which encoding is not narrowly focused on order information (Nairne et al., 1991).

For free recall, the analysis revealed four significant effects. First, the effect of encoding condition $[F(1,47)=$ $11.28, M S_{\mathrm{e}}=0.1367$ ] indicates better recall for the read than for the generate items. Second, the effect of item type $\left[F(1,47)=122.04, M S_{\mathrm{e}}=0.3107\right]$ indicates better recall for words than for nonwords. The third effect is that of serial position $\left[F(7,329)=26.56, M S_{\mathrm{e}}=0.0581\right]$, and the fourth was the item type $\times$ serial position interaction $\left[F(7,329)=5.37, M S_{\mathrm{e}}=0.0584\right]$, which indicates that the recall advantage for words was greater at earlier serial positions. No other effects were significant $\left(F_{\mathrm{S}}<1\right)$. The present results replicate and extend Nairne et al. (1991) in finding a reverse generation effect for both words and 


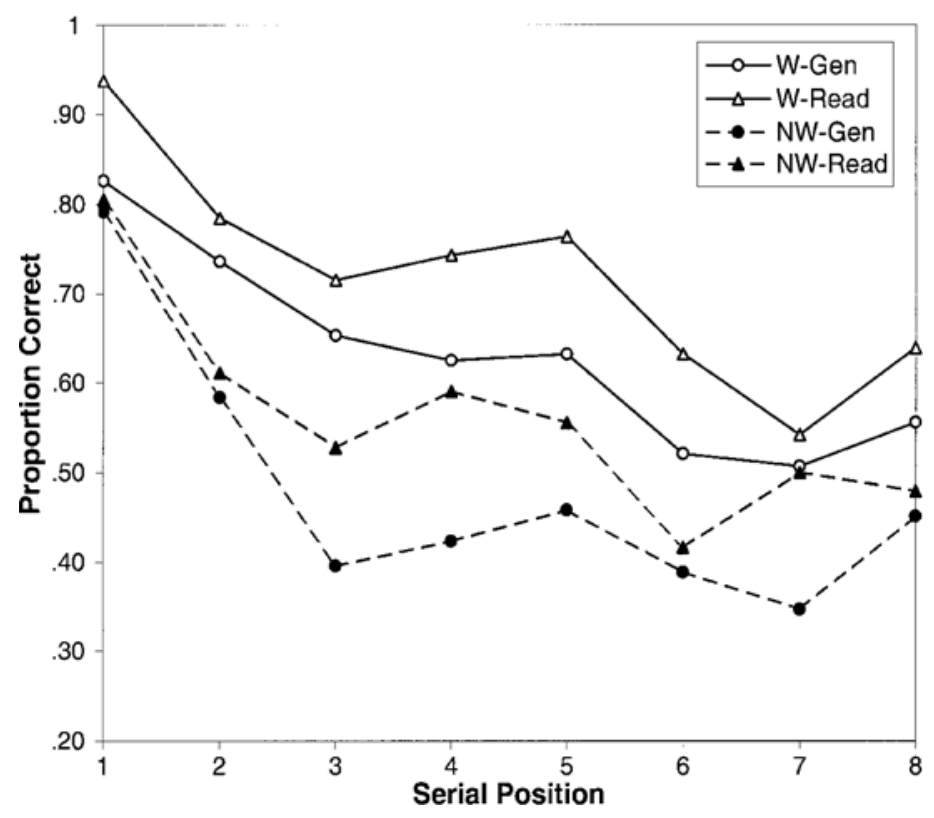

Figure 2. Experiment 2: Mean order reconstruction scores as a function of item type, encoding condition, and serial position $(\mathrm{W}=$ word, $\mathrm{NW}=$ nonword, Gen $=$ generate condition).

nonwords. This is exactly what is expected if free recall is especially reliant on order information in the present paradigm (DeLosh \& McDaniel, 1996; Nairne et al., 1991; Serra \& Nairne, 1993).

To assess the use of order information in recall, the index of Asch and Ebenholtz (1962) is typically used (e.g.,
Burns et al., 1993; DeLosh \& McDaniel, 1996; Nairne et al., 1991). This index assesses the extent to which relative serial order at study is preserved in recall order, with a value of .50 as chance correspondence. Because the Asch-Ebenholtzindex requires the recall of more than one item on at least one of the recall tests, it could not be com-

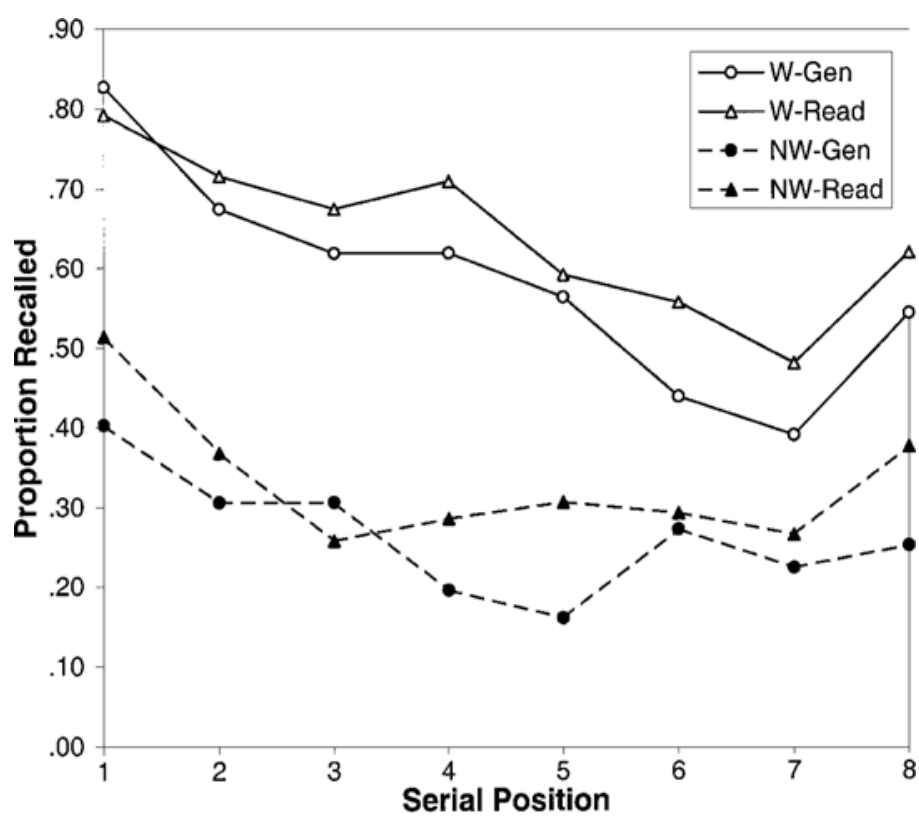

Figure 3. Experiment 2: Mean recall as a function of item type, encoding condition, and serial position $(\mathrm{W}=$ word, $\mathrm{NW}=$ nonword, $\mathrm{Gen}=$ generate condition). 
puted for all participants in all conditions (i.e., some participants failed to recall more than a single item from each study list of a given condition). For words, the index was computed for 45 participants, yielding means of .82 and .72 for the read and generate conditions, respectively. For nonwords, the index was computed for 41 participants, yielding means of .79 and .65 for the read and generate conditions, respectively. All values are significantly above chance $(t \mathrm{~s}>3.00)$, consistent with the notion that serialorder information was used during recall (e.g., Nairne et al., 1991). Forty of the participants produced valid values for the index in each condition. For these participants, a 2 (encoding condition) $\times 2$ (item type) ANOVA revealed a significant effect of encoding condition $[F(1,39)=$ 13.75, $\left.M S_{\mathrm{e}}=0.0426\right]$, indicating that the index was higher in the read than generate condition. No other effect was significant ( $p$ s $<.10$ ). Likewise, a comparison restricted to the nonwords indicated a significant effect of encoding condition $[t(39)=2.51]$. This converges with the results of the order reconstruction test in indicating a deficit in order encoding in the generate condition for both words and nonwords.

\section{EXPERIMENT 3}

The results Experiment 1 imply that the aspects of generation responsible for enhanced item encoding can be dissociated from those aspects responsible for disrupted order encoding. Experiment 3 extends this result to a different type of unfamiliar stimulus, unfamiliar word compounds. Gardiner and Hampton (1985) had participants generate or read word compounds that were familiar (e.g., cheese cake) or unfamiliar (e.g., tomato cake). On a recall test for the word compounds, a generation advantage was found for the familiar compounds but not for the unfamiliar compounds. This limitation on the generation effect bears an interesting relationship to the limitation found with nonwords. In contrast with nonwords, unfamiliar compounds are composed of familiar words with preexisting lexical and semantic representations. However, as a unit, the compounds do not correspond to any preexisting, unitized representation. Consequently, contrasting familiar and unfamiliar word compounds makes an interesting test case to determine whether the item-enhancing effects of generation can again be dissociated from the order-disrupting effects of generation.

Experiment 3 also serves two other goals. First, Gardiner and Hampton (1985) assessed memory with a recall test that is typically viewed as less sensitive to item encoding than are recognition tests (e.g., Engelkamp \& Zimmer, 1994; Hunt \& McDaniel, 1993). The use of a recognition test provides a stronger assessment of whether item memory is enhanced when familiar but not unfamiliar compounds are generated. Second, virtually all of the prior research on the effects of generation on order memory have used individual words as stimuli (e.g., Burns, 1996; Burns et al., 1993; Greene et al., 1998; Nairne et al., 1991; Serra \& Nairne, 1993), the exception being Greene et al. (1998, Experiment 2), who used numbers. These studies have generally found that generating familiar words enhances item memory but disrupts order memory. In the present study, the results of the familiar compounds will determine whether this result generalizes to stimuli consisting of multiple words.

\section{Method}

Participants. Forty-eight undergraduates at Southern Methodist University participated in exchange for course credit in psychology courses.

Materials and Design. Compound type (familiar vs. unfamiliar), encoding condition (read vs. generate), and test type (order reconstruction vs. recognition) were varied within subjects. The critical study materials consisted of 112 familiar compound words and two-word phrases (e.g., cheese cake, home run, mug shot, door $k n o b$ ), some of which were selected from published sources (Inhoff, Briihl, \& Schwartz, 1996; Reinitz \& Demb, 1994), and the rest were developed by the experimenter. The compounds were randomly organized into 16 lists of seven items each and were used to create two master sets. The first set was created by randomly assigning half of the lists to appear in the unfamiliar condition. Unfamiliar compounds were created by swapping the second word of each compound across two lists designated to be in the unfamiliar condition. This scheme ensured that no familiar compound could be formed by combining words across items within an unfamiliar list. The resulting compounds were examined to be sure that no familiar compounds or phrases were inadvertently produced. The second master set was created by assigning the other half of the lists (those in the familiar condition in the first set) to be in the unfamiliar condition and by creating unfamiliar compounds in the same manner. Next, two versions of each master set were created to counterbalance the assignment of lists to the two encoding conditions (read and generate). Thus, all compounds within a study list were of the same type (either familiar or unfamiliar) and were presented in the same encoding condition (either read or generate). Compound type and encoding condition varied across study lists. The four versions of the study materials consisted of the same set of 224 individual words, and across participants, these words appeared equally often in read and generated, familiar and unfamiliar compounds. Furthermore, each of these four versions formed the basis of two sets of study-test blocks, across which each study list was followed once by the orderreconstruction test and once by asterisks. The study-test blocks were randomly sequenced, subject to the constraint that no more than two consecutive study lists could be of the same compound type, encoding condition, or test type.

The critical blocks were preceded by two practice blocks. None of the words in the practice lists duplicated words in the critical lists. The end-of-session recognition test consisted of 56 studied compounds ( 14 of each of the compound-type-encoding condition combinations) from the untested blocks. The studied compounds were randomly intermixed with 32 new compounds, of which 16 were familiar and 16 unfamiliar. The new compounds consisted of words not previously seen in the experimental context.

Procedure. The procedures were the same as in Experiment 1, with the following alterations. The first part of the experiment consisted of 18 ( 2 practice and 16 critical) study-test blocks. Each study list consisted of seven compounds. In the generate condition, the two words of the compound were presented on the computer screen underlined, in reversed order, and with a single space between them. The participants were instructed to reverse the order of the underlined words, write the resulting compound on the answer sheet, and to try to remember it for a later memory test. The following instructions appeared on the bottom of the screen: Reverse the order of the words to form the study pair. In the read condition, the two words were presented in the correct order and were not underlined. The 
Table 2

Experiment 3: Proportion of Items Judged as Old on the Recognition Memory Test as a Function of Encoding Condition and Compound Type

\begin{tabular}{cccc}
\hline & \multicolumn{3}{c}{ Encoding Condition } \\
\cline { 2 - 4 } Compound Type & Read & Generate & New \\
\hline Familiar & .71 & .82 & .14 \\
Unfamiliar & .64 & .65 & .12 \\
\hline
\end{tabular}

participants were instructed to simply copy these compounds onto the answer sheet in the order in which they appeared and to try to remember them for the subsequent memory test. The following reminder appeared on the bottom of the screen: Copy the words in the order that they appear. For both encoding conditions, the stimuli and instructions were presented for $5,900 \mathrm{msec}$ followed by a blank screen for $100 \mathrm{msec}$.

The distractor task and the test phase for untested lists (asterisks blocks) were identical to those in Experiment 1. The orderreconstruction task was also the same, with the exception that each compound occupied a separate line on the computer screen. The constituent words of each compound were separated by a space.

As in Experiment 1, the last study-test block was followed by the end-of-session recognition test. The participants were told that their memory for the compounds would be tested. It was made clear that the test items would be presented in the form in which they had been written down on the answer sheet-that is, with the words reversed for the generated compounds or in the original order for the read compounds. Each test compound was presented individually on the computer screen, with the constituent words separated by a space. The test compound remained on the screen until the participant responded.

\section{Results and Discussion}

During presentation of the study lists, the participants correctly generated $99.3 \%$ and $98.1 \%$ of the familiar and unfamiliar compounds, respectively. The corresponding means for the read items were $100 \%$ and $99.7 \%$.

The results of the recognition test (Table 2) are considered first. A preliminary analysis indicated that false alarm rates did not differ between familiar and unfamiliar compounds $[F=1.56]$. $d^{\prime}$ recognition accuracy was submitted to a $2 \times 2$ ANOVA, with compound type and encoding condition as factors. All three effects were significant: compound type $\left[F(1,47)=6.81, M S_{\mathrm{e}}=0.5230\right]$, which indicated better recognition of familiar than of unfamiliar compounds, encoding condition $\left[F(1,47)=9.69, M S_{\mathrm{e}}=\right.$ 0.2338 , which indicated higher performance in the generate than in the read condition, and the interaction $[F(1,31)=$ $\left.17.21, M S_{\mathrm{e}}=0.1045\right]$. Planned contrasts clarify the interaction, indicating an effect of encoding condition for familiar compounds $[t(47)=4.78$; mean $d \mathrm{~s}=1.90$ and 2.31 for the read and generate conditions, respectively] but not for unfamiliar compounds $[t(47)<1$; mean $d$ s $=$ 1.82 and 1.85 for the read and generate conditions, respectively]. The same pattern of results was obtained using corrected hits (hits - false alarms) as the measure of accuracy.

The recognition results indicate a generation effect in recognition for familiar but not for unfamiliar compounds. This provides a conceptual replication of Gardiner and
Hampton's (1985) results. In Gardiner and Hampton's study, participants either read compounds or generated them from short definitions (e.g., a cake made of cheese, a cloth for the hair), and memory was tested with recall. In the present study, a generation task quite similar to the letter transposition task of Experiments 1 and 2 was used and tested memory with recognition because of its greater sensitivity to item encoding. Despite these differences, the same results obtained. These results are consistent with the view that generation enhances the amount of item encoding for familiar compounds but not for unfamiliar compounds, presumably because the latter have no preexisting unitized representation at the level of the compound (Gardiner \& Hampton, 1985).

For the unfamiliar compounds, the power to detect a generation effect the size of that found with familiar compounds $(d=.99)$ exceeds .99 . For a to-be-detected effect, one-third smaller than that found with familiar compounds $(d=.66)$, the power is .94 . Thus, the present experiment had substantial power to detect an effect of generation on recognition memory for the unfamiliar compounds.

The order results (Figure 4) are quite similar to those of Experiments 1 and 2: Generation disrupted order memory for both familiar and unfamiliar compounds. The proportions correct were submitted to a $2 \times 2 \times 7$ ANOVA, with compound type, encoding condition, and serial position as factors. The analysis revealed significant main effects of compound type $\left[F(1,47)=9.66, M S_{\mathrm{e}}=0.2200\right]$, encoding condition $\left[F(1,47)=9.93, M S_{\mathrm{e}}=0.1985\right]$, and serial position $\left[F(6,282)=15.28, M S_{\mathrm{e}}=0.0775\right]$. None of the interactions were significant (all $\left.F_{\mathrm{S}}<1\right)$.

The effect of item type indicates that order memory was better for familiar than for unfamiliar compounds, similar to the finding that words produced better order memory than did nonwords. The critical result is the main effect of encoding condition, which indicates better order memory for the read than for the generate items. Averaged over serial positions, the mean proportions correct for read and generated familiar compounds were .70 and .61 , respectively. The corresponding means for unfamiliar compounds were .61 and .54 [an analysis restricted to the unfamiliar compounds also indicated a significant negative generation effect; $\left.F(1,47)=4.71, M S_{\mathrm{e}}=0.1450\right]$. There are two aspects of this result to highlight. First, in combination with the recognition results, the results of the familiar compounds extend the generality of earlier research demonstrating that generation enhances item memory and disrupts order memory (e.g., Burns, 1996; Burns et al., 1993; Nairne et al., 1991; Serra \& Nairne, 1993). The present results indicate that such a pattern is obtained with word compounds as well as with individual words. Second, and consistent with Experiment 1, a condition that did not give rise to enhanced item encoding still produced a negative generation effect on order memory. Generation of unfamiliar compounds did not enhance item recognition and yet it produced a decrement in order memory of a comparable magnitude as that produced by the generation of familiar compounds. 


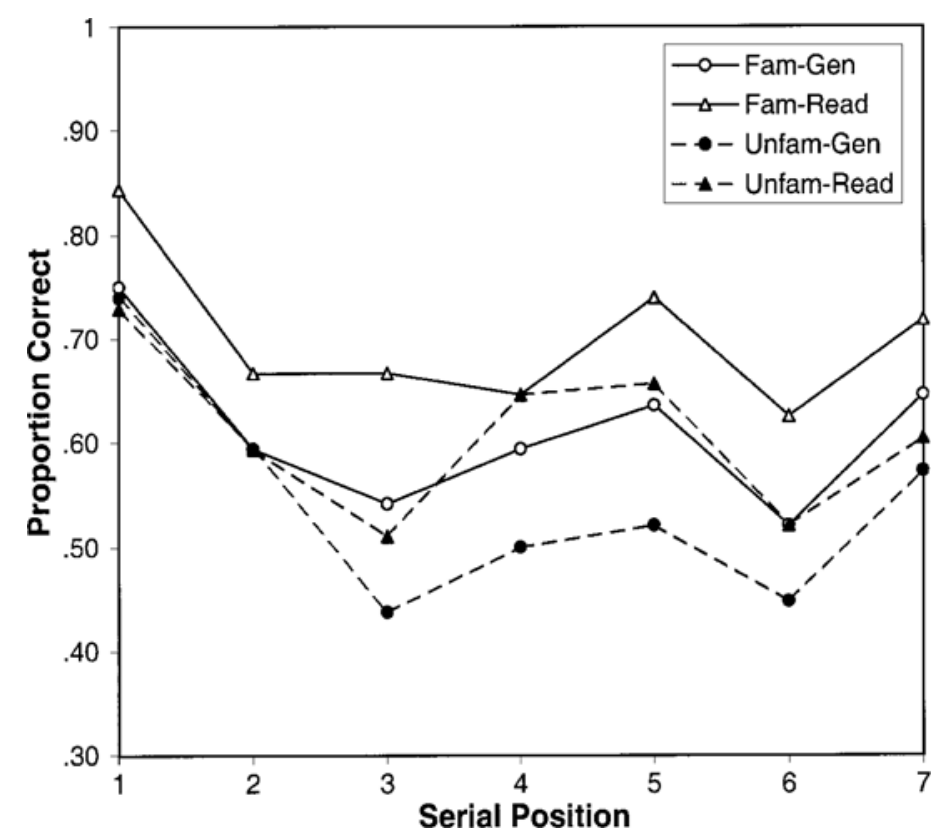

Figure 4. Experiment 3: Mean order reconstruction scores as a function of compound type, encoding condition, and serial position (Fam = familiar compound, Unfam = unfamiliar compound, Gen = generate condition).

\section{GENERAL DISCUSSION}

A number of encoding manipulations (generation, perceptual identification, word frequency, bizarre imagery, self-enactment) have been found to produce opposite effects on item and order memory (e.g., Burns, 1996; DeLosh \& McDaniel, 1996; Engelkamp \& Dehn, 2000; McDaniel et al., 1995; Mulligan, 1999; Nairne et al., 1991; Serra \& Nairne, 1993), suggesting an intimate relationship between enhanced item encoding and disrupted order memory. Three experiments investigated this relationship with the generation of familiar and unfamiliar stimuli. With regard to familiar stimuli, the present results replicate and extend prior research. In Experiment 1, generating words enhanced item recognition but disrupted order memory, consistent with earlier research (Nairne et al., 1991; Serra \& Nairne, 1993). In Experiment 3, this pattern of results was extended to multiple word units: Generating familiar word compounds likewise enhanced item memory and disrupted order reconstruction. In addition, when order information was an important determinant of recall (Experiment 2), generation disrupted free recall as well as order memory, a result previously found for words (e.g., Nairne et al., 1991) is now also demonstrated with nonwords. More important, however, were the results obtained for unfamiliar stimuli. Generating nonwords or unfamiliar word compounds produced no enhancement in item memory (as measured by recognition) but disrupted order memory to the same extent as the generation of familiar stimuli. Thus, the item-enhancing effects of gener- ation were dissociated from its disruptive effect on order memory.

These results are similar to the results of Mulligan (2000a), reviewed in the introduction, who found that delaying the mask in the perceptual interference manipulation eliminated the typical item enhancement, but persisted in disrupting order memory. A converging result was reported by Mulligan (2001b), who examined the effects of word frequency on item and order memory. It has previously been noted that word frequency can produce opposite effects on item and order memory; low-frequency words produce superior recognition, whereas high-frequency words produce superior order memory (e.g., DeLosh \& McDaniel, 1996). Mulligan (2001b) contrasted high-, low-, and very low-frequency words on recognition memory and order memory. Compared to high-frequency words, low-frequency words enhanced recognition, whereas very low-frequency words did not. Both low- and very low-frequency words, however, produced worse memory for order. This implies that the item-enhancing effects of lower word frequency may be dissociated from its orderdisrupting effects. The present results join these previous results in documenting versions of the perceptual interference, word frequency, and generation manipulations that fail to enhance item memory while disrupting order memory.

Next, we consider some of the theoretical implications of these results. The order-encoding hypothesis has been put forth as a unifying theoretical view to account for the set of variables, described above, that both enhance item 
memory and disrupt order memory (e.g., DeLosh \& McDaniel, 1996; Engelkamp \& Dehn, 2000; McDaniel et al., 1995; Mulligan, 1999; Nairne et al., 1991; Serra \& Nairne, 1993; see discussion in McDaniel et al., 2000). This view proposes that the more unusual encoding condition (e.g., the generate condition, bizarre sentences, low-frequency words) attracts or requires more attentional resources for processing and interpreting the item than does the more usual condition (e.g., the read condition, common sentences, high-frequency words). According to this view, this simultaneously enhances encoding of item features and draws resources away from the encoding of serial order (and other relational) information in the unusual condition. More generally, under this view, those aspects of a manipulation that enhance item encoding are assumed to be the same as those that disrupt order encoding.

The present results provide a challenge to the orderencoding hypothesis because this hypothesis depicts disrupted order encoding as a by-product of enhanced item encoding. Consequently, it is not expected that disrupted order encoding could be dissociated from enhanced item encoding as in the present results. Given the general success of the order-encoding hypothesis, however, it is important to consider variants of the order-encoding hypothesis that might be applied in the present case. First, it could be argued that nonwords and unfamiliar word compounds are sufficiently unusual in their own right that encoding resources are drawn to their item characteristics regardless of whether they are presented in the generate or read condition. This would account for the null effect of generation on recognition. However, this view implies no functional difference between generating and reading unfamiliar stimuli, an implication contradicted by the effect of generation on order memory and free recall (Experiment 2). Generation does have an effect on unfamiliar stimuli but one that, in the present case, is not manifest in recognition.

Second, it might be argued that unfamiliar (meaningless) materials induce qualitatively different processing than do familiar (meaningful) materials (cf. Auble \& Franks, 1978). With familiar materials, the tradeoff between item and order encoding may well hold. For unfamiliar material, the analysis and interpretation of the item may be attenuated because there is no meaningful representation to guide processing. Consequently, there may be no difference in item processing between the read and generate conditions. Under this view, the order-encoding hypothesis holds for familiar materials but does not generalize to unfamiliar materials. This in turn suggests that enhanced item encoding might not be necessary for disrupted order encoding (because it occurs for unfamiliar stimuli) but it may be sufficient. This is an interesting idea, worth considering. However, it lacks parsimony. The experimental results indicate that generation disrupts order memory for both familiar and unfamiliar stimuli, but this theoretical view suggests that the disruption occurs for different reasons. For familiar stimuli, the disrupted order encoding is a by-product of differential item encoding, whereas for unfamiliar stimuli, it is not.

Third, one might argue that generation induces an encoding tradeoff between item and order information for both familiar and unfamiliar items, but that in the case of unfamiliar stimuli, the item representations are not available or are insufficient to render the stimulus distinctive. This view is consistent with those arguing that (1) the generation effect is due to enhanced encoding of item characteristics that render the generated item relatively distinctive (e.g., deWinstanley et al., 1996; Hirshman \& Bjork, 1988; Hunt \& McDaniel, 1993; McDaniel et al., 1988; Steffens \& Erdfelder, 1998) and (2) the generated stimulus must possess a preexisting, unitized representation for generation to produce distinctiveness (Gardiner et al., 1988; Gardiner \& Hampton, 1985; McElroy \& Slamecka, 1982; Nairne et al., 1985; Payne et al., 1986). ${ }^{2}$ This version of the order-encoding view would argue that generating an item attracts attention to the item characteristics and away from representations of serial order, but in the case of unfamiliar stimuli, the item representations are not sufficient to engender distinctiveness (and thus no generation effect ensues). Thus, any enhanced item processing in the generation condition would be ineffective for later recognition. Under this view, the dissociations obtained in the present study imply different representational bases for item and order memory but do not necessarily imply different encoding mechanisms or processes. ${ }^{3}$

Although this elaboration of the order-encoding view is consistent with the present results, it does not apply more broadly. The order-encoding view was designed to apply to an array of encoding manipulations that produce a common pattern of enhanced item memory and disrupted order memory (DeLosh \& McDaniel, 1996). However, the present extension of the order-encoding process does not apply to the results of the perceptual-interference manipulation (Mulligan, 2000a), which is among the manipulations encompassed by the order-encoding hypothesis (Engelkamp \& Dehn, 2000; McDaniel et al., 2000). Delaying the mask in the perceptual-interference condition eliminates the perceptual-interference effect in item memory but produces as large a disruption of order memory as the standard perceptual-interference condition (Mulligan, 2000a). Importantly, these results occurred for familiar words. Thus, the lack of enhanced item memory cannot be attributed to the use of materials without preexisting representations; the very same words that produced enhanced item memory in the standard perceptualinterference condition failed to do so in the delayed-mask condition. Consequently, the results of the perceptualinterference manipulation resist interpretation in terms of an encoding tradeoff within a common encoding mechanism.

Mulligan (2000a) interpreted these results in terms of the compensatory-processing account of the perceptualinterference effect, an account that proposes that perceptual interference enhances item memory during initial per- 
ception of the stimulus and disrupts interitem relational processing postperceptually. The distinction between perceptual and postperceptual encoding in this account is similar to the distinction between initial interpretative encoding versus later elaborative encoding (Masson \& MacLeod, 1992,1997) or generative versus post-generative encoding (Burns, 1992) used in accounts of the generation effect. This type of distinction may be important if a general account of item-order dissociations is to be applied across a range of encoding manipulations.

\section{REFERENCES}

Asch, S. E., \& Ebenholtz, S. M. (1962). The process of free recall: Evidence for nonassociative factors in acquisition and retention. Journal of Psychology, 54, 3-31.

Auble, P. M., \& Franks, J. J. (1978). The effects of effort toward comprehension on recall. Memory \& Cognition, 6, 20-25.

BURNS, D. J. (1992). The consequences of generation. Journal of Memory \& Language, 31, 615-633.

Burns, D. J. (1996). The item-order distinction and the generation effect: The importance of order information in long-term memory. American Journal of Psychology, 109, 567-580.

Burns, D. J., Curti, E. T., \& Lavin, J. C. (1993). The effects of generation on item and order retention in immediate and delayed recall Memory \& Cognition, 21, 846-852.

CoHEN, J. (1988). Statistical power analysis for the behavioral sciences. Hillsdale, NJ: Erlbaum.

DeLosh, E. L., \& McDaniel, M. A. (1996). The role of order information in free recall: Application to the word-frequency effect. Journal of Experimental Psychology: Learning, Memory, \& Cognition, 22, 1136-1146.

DeWinstanley, P. A., Bjork, E. L., \& Bjork, R. A. (1996). Generation effects and the lack thereof: The role of transfer-appropriate processing. Memory, 4, 31-48.

EngelKamp, J., \& Dehn, D. A. (2000). Item and order information in subject-performed tasks and experimenter-performed tasks. Journal of Experimental Psychology: Learning, Memory, \& Cognition, 26, 671-682.

Engelkamp, J., \& Zimmer, H. D. (1994). The human memory: A multimodal approach. Seattle: Hogrefe \& Huber.

Gardiner, J. M., GregG, V. H., \& Hampton, J. A. (1988). Word frequency and generation effects. Journal of Experimental Psychology: Learning, Memory, \& Cognition, 14, 687-693.

Gardiner, J. M., \& Hampton, J. A. (1985). Semantic memory and the generation effect: Some tests of the lexical activation hypothesis. Journal of Experimental Psychology: Learning, Memory, \& Cognition, 11, 732-741.

Greene, R. L. (1992). Human memory: Paradigms and paradoxes. Hillsdale, NJ: Erlbaum.

Greene, R. L., Thapar, A. \& Westerman, D. L. (1998). Effects of generation on memory for order. Journal of Memory \& Language, $\mathbf{3 8}$ 255-264.

Hirshman, E., \& BJork, R. A. (1988). The generation effect: Support for a two-factor theory. Journal of Experimental Psychology: Learning, Memory, \& Cognition, 14, 484-494.

Hirshman, E., \& Mulligan, N. W. (1991). Perceptual interference improves explicit memory but does not enhance data-driven processing. Journal of Experimental Psychology: Learning, Memory, \& Cognition, 17, 507-513.

Hirshman, E., Trembath, D., \& Mulligan, N. W. (1994). Theoretical implications of the mnemonic benefits of perceptual interference. Journal of Experimental Psychology: Learning, Memory, \& Cognition, 20, 608-620.

Hunt, R. R. \& McDaniel, M. A. (1993). The enigma of organization and distinctiveness. Journal of Memory \& Language, 32, 421-445.

InHoff, A. W., BriIhl, D., \& Schwartz, J. (1996). Compound word effects differ in reading, on-line naming, and delayed naming tasks. Memory \& Cognition, 24, 466-476.
Johns, E. E., \& Swanson, L. C. (1988). The generation effect with nonwords. Journal of Experimental Psychology: Learning, Memory, \& Cognition, 14, 180-190.

Kelley, M. R., \& NAIRNE, J. S. (2001). von Restorff revisited: Isolation, generation, and memory for order. Journal of Experimental Psychology: Learning, Memory, \& Cognition, 27, 54-66.

KInOSHITA, S. (1989). Generation enhances semantic processing? The role of distinctiveness in the generation effect. Memory \& Cognition, $17,563-571$

KuČERA, H., \& FrANCIS, W. N. (1967). Computationalanalysis of presentday American English. Providence, RI: Brown University Press.

Masson, M. E. J., \& MacLeod, C. M. (1992). Reenacting the route to interpretation: Enhanced perceptual identification without prior perception. Journal of Experimental Psychology: General, 121, 145-176.

Masson, M. E. J., \& MACLEod, C. M. (1997). Episodic enhancement of processing fluency. In D. L. Medin (Ed.), The psychology of learning and memory (Vol. 37, pp. 155-210). San Diego: Academic Press.

McDaniel, M. A., DeLosh, E. L., \& Merritt, P. S. (2000). Order information and retrieval distinctiveness: Recall of common versus bizarre material. Journal of Experimental Psychology: Learning, Memory, \& Cognition, 26, 1045-1056.

McDaniel, M. A., Einstein, G. O., DeLosh, E. L., May, C. P., \& BRADY, P. (1995). The bizarreness effect: It's not surprising, it's complex. Journal of Experimental Psychology: Learning, Memory, \& Cognition, 21, 422-435.

McDaniel, M. A., Wadill, P. J., \& Einstein, G. O. (1988). A contextual account of the generation effect: A three-factor theory. Journal of Memory \& Language, 27, 521-536.

McElroy,L. A., \& SLAmeCKa, N. J. (1982). Memorial consequences of generating nonwords: Implications for semantic-memory interpretations of the generation effect. Journal of Verbal Learning \& Verbal Behavior, 21, 243-259.

Mulligan, N. W. (1996). The effects of perceptual interference at encoding on implicit memory, explicit memory, and memory for source. Journal of Experimental Psychology: Learning, Memory, \& Cognition, 22, 1067-1087.

Mulligan, N. W. (1998). Perceptual interference at encoding enhances recall for high- but not low-imageability words. Psychonomic Bulletin \& Review, 5, 464-469.

Mulligan, N. W. (1999). The effects of perceptual interference at encoding on organization and order: Investigating the roles of itemspecific and relational information. Journal of Experimental Psychology: Learning, Memory, \& Cognition, 25, 54-69.

Mulligan, N. W. (2000a). Perceptual interference and memory for order. Journal Memory \& Language, 43, 680-697.

Mulligan, N. W. (2000b). Perceptual interference at encoding enhances item-specif ic encoding and disrupts relational encoding: Evidence from multiple recall tests. Memory \& Cognition, 28, 539-546.

Mulligan, N. W. (2001a). Generation and hypermnesia. Journal of Experimental Psychology: Learning, Memory, \& Cognition, 27, 436-450.

Mulligan,N. W. (2001b). Word frequency and memory: Effects on absolute versus relative order memory and on item memory versus order memory. Memory \& Cognition, 29, 977-985.

NAIRNE, J. S. (1988). The mnemonic value of perceptual identification. Journal of Experimental Psychology: Learning, Memory, \& Cognition, 14, 244-255.

NAirne, J. S., Pusen, C., \& Widner, R. L. JR. (1985). Representation in the mental lexicon: Implications for theories of the generation effect. Memory \& Cognition, 13, 183-191.

Nairne, J. S., Reigler, G. L., \& SERra, M. (1991). Dissociative effects of generation on item and order retention. Journal of Experimental Psychology: Learning, Memory, \& Cognition, 17, 702-709.

NAIRnE, J. S., \& Widner, R. L. (1987). Generation effects with nonwords: The role of test appropriateness. Journal of Experimental Psychology: Learning, Memory, \& Cognition, 13, 164-171.

Payne, D. G., Neely, J. H. \& Burns, D. J. (1986). The generation effect: Further tests of the lexical activation hypothesis. Memory \& Cog nition, 14, 246-252.

ReInitz, M. T., \& Demb, J. B. (1994). Implicit and explicit memory for compound words. Memory \& Cognition, 22, 687-694.

SerRA, M., \& NAIRNE, J. S. (1993). Design controversies and the gen- 
eration effect: Support for an item-order hypothesis. Memory \& Cognition, 21, 34-40.

Slamecka, N. J., \& GRAF, P. (1978). The generation effect: Delineation of a phenomenon. Journal of Experimental Psychology: Human Learning \& Memory, 4, 592-604.

SlamecKa, N. J., \& Katsaiti, L. T. (1987). The generation effect as an artifact of selective displaced rehearsal. Journal of Memory \& Language, 26, 589-607.

Steffens, M. C., \& Erdfelder, E. (1998). Determinants of positive and negative generation effects in free recall. Quarterly Journal of Experimental Psychology, 51A, 705-733.

\section{NOTES}

1. The findings of Greene, Thapar, and Westerman (1998) indicate that this result does not apply to tests requiring judgments of relative recency, which produce equivalent performance for read and generate conditions (Greene et al., 1998; see Mulligan, 2000a, for a similar result). This implies that tests of absolute order (e.g., order reconstruction) and relative recency might rely on somewhat different memory processes. Because the present article focuses on memory for absolute order and because the vast majority of relevant prior research has used tests of absolute order, subsequent use of the term order memory refers only to memory for absolute order.

2 . This view should be restricted to memory tests in which the entire stimulus (e.g., nonword or unfamiliar compound) is the basis of retrieval. Nairne and Widner (1987) reported a generation effect with nonwords when the retrieval task focused only on the parts of the letter string manipulated in the generation task.
3. It should be noted that McDaniel et al. (2000) recently explored another alternative to the order-encoding hypothesis. They examined the bizarre-imagery effect in recall and its reliance on experimental design; like the generation effect, the bizarre-imagery effect is typically found in mixed-list but not pure-list designs. As an alternative to the order-encoding hypothesis, McDaniel et al. (2000) considered (and found support for) a retrieval-based hypothesis, which argues that a mixed-list design provides a retrieval context favoring the unusual items, whereas pure lists do not. In this retrieval based account, the item advantage of the bizarre items is conceived of as a retrieval phenomenon rather than as an encoding phenomenon. Thus, one could argue that in a general sense, this account is consistent with the present results because it raises the possibility that enhanced item distinctiveness arises via a different mnemonic mechanism than does the disruption of order memory. However, two points should be noted. First, the retrieval-based account was specifically developed to account for recall results rather than to provide a complete account of potential tradeoffs in item and order memory. Consequently, it does not provide a detailed account of the present results. Second, this model derived from research on the bizarre-imagery effect and may not generalize to the generation effect (McDaniel et al., 2000, p. 1053). Application of this model to the generation effect and further specification of this account, specifically in terms of order memory, are likely to be very fruitful areas for additional research.

(Manuscript received November 5, 2001; revision accepted for publication May 9, 2002.) 